\title{
Security performance analysis for power domain NOMA employing in cognitive radio networks
}

\author{
Thi-Anh Hoang, Chi-Bao Le, Dinh-Thuan Do \\ Industrial University of Ho Chi Minh City, Vietnam
}

\begin{abstract}
Article Info
ABSTRACT

Article history:

Received May 16, 2019

Revised Sep 26, 2019

Accepted Feb 1, 2020

\section{Keywords:}

Cognitive radio

Non-orthogonal multiple access

(NOMA)

Physical-layer security

The power domain non-orthogonal multiple access (NOMA) technique introduces one of the fundamental characteristics and it exhibits the possibility of users to decode the messages of the other paired users on the same resources. In cognitive radio inspired NOMA (CR-NOMA), the base station (BS) has to serve untrusted users or users with different security clearance. This phenomenon raises a security threat particularly in such CR-NOMA. This paper develops a tractable analysis framework to evaluate the security performance of cooperative non-orthogonal multiple access (NOMA) in cognitive networks, where relay is able to serve two far NOMA users in the presence of external eavesdropper. In particular, we study the secrecy outage probability in a two-user NOMA system. This situation happens in practical the BS is pairing a legitimate user with another untrusted user. Main reason is that the non-uniform distribution in terms of trusted and untrusted users in the cell. By performing numerical results demonstrate the performance improvements of the proposed NOMA scheme in comparison to that of several situations in terms of different parameters. Furthermore, the security performance of NOMA is shown to verify the derived expressions.
\end{abstract}

This is an open access article under the $\underline{C C B Y-S A}$ license.

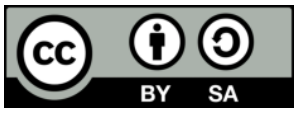

\section{Corresponding Author:}

Dinh-Thuan Do,

Industrial University of Ho Chi Minh City,

12 Nguyen Van Bao, Go Vap, Ho Chi Minh City, Vietnam.

Email: dodinhthuan@iuh.edu.vn

\section{INTRODUCTION}

As a promising multiple access (MA) technique, Non-orthogonal multiple access (NOMA) has been proposed to adapt the challenging requirements of the future mobile communication networks. Several strict requirements in $5 \mathrm{G}$ wireless communication can be achieved such as high data speed, spectral efficiency, massive connectivity and low latency [1-10]. In [11, 12] the NOMA can be integrated with green communication to enable ability of wireles s power transfer in cooperative NOMA network. Other application of device-to-device (D2D) to NOMA is introduced as in [13]. Different with the conventional MA techniques, the power domain is used to serve a multiple user in NOMA at different power levels and such paradigm exhibits a high spectral efficiency [14]. To permit the unlicensed users operating on the spectrum allocated for the licensed users, cognitive radio networks (CRNs) have been recommended to achieve full advantage of radio resource. In these situations, the quality of service of the primary user networks is maintained [15, 16]. In general, power domain multiple access scheme and spectrum efficiency are advantages are able to achieved. Hence, by introducing NOMA to PUNs, the system performance of cognitive radio can be further increased. 
In the other trend, dedicated relays [17-19] are also deployed to reform cooperative NOMA transmission [20]. To enhance the reliability of the NOMA-weak users, [21] adopted a dedicated relay network. Also, with the help of a relay (both AF and DF protocols) [22] presented a secrecy analys is of a NOMA system. The other works reported in [23-25], in which the CR networks are studied with an underlay paradigm. From raised analytical results according to the previous analysis, the improved system performance benefits from combining system between overlay CR and NOMA. Unfortunately, overlay $\mathrm{CR}$ can enhance system performance and also produce the risk of infection of illegal information simultaneously. On the contrary, NOMA can achieve better secrecy performance than OMA [22]. The physical layer security performance in overlay CR-NOMA networks is still a challenging issue and it motivates this study. This paper determines physical layer security (PLS) in cognitive radio inspired non-orthogonal multiple access (CRNOMA) networks with secondary source and multiple secondary users under impact of primary destination.

To manage the interference among the users and guarantee the quality of services (QoS) of primary users, a new secure NOMA transmission strategy is designed, where the primary and secondary users are paired according to their channel gains, respectively, and power-domain NOMA is employed to transmit the signal. This paper studies a downlink cooperative CR-NOMA model with interference temperature constraint (ITC) at the primary receiver. Exact closed-form expressions for the OP of the far NOMA users are derived. These expressions are verified by Monte-Carlo simulations.

\section{SYSTEM MODEL}

A downlink cooperative underlay CR-NOMA is considered in this study. In this model, secondary network (SN) consists of a secondary base station (S), a relay (R) and two far NOMA users (D1; D2) can operate together with primary network containing primary destination (PD) who make impact on system performance at secondary network, as shown in Figure 1. Regarding secure performance, existence of eavesdropper E need be concerned. The links are as signed channel as in Figure 1 and these channels follow flat Rayleigh fading model. $h_{S R}$ is the channel between BS and relay, $h_{S R}$ is the channel between Relay and two users. $P_{S}$ is transmit power at the BS. We denote the superimposed signal to transmit from the BS to secondary destinations $\sqrt{a_{1} P_{S}} x_{1}+\sqrt{a_{2} P_{S}} x_{2}$. While $a_{1}, a_{2}$ are power allocation factors in NOMA satisfying $a_{1}+a_{2}=1,\left(a_{1}>a_{2}\right)$.

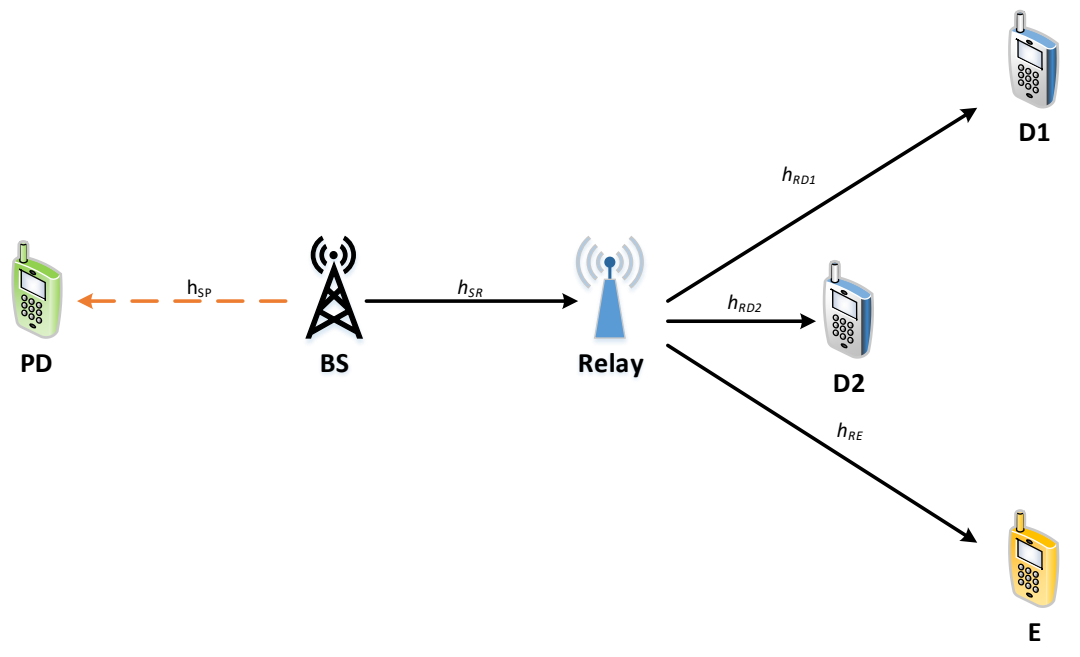

Figure 1. System model of secure CR-NOMA

The transmit power constraint is determined at the BS:

$$
P_{S} \leq \min \left(\frac{I}{\left|h_{S P}\right|^{2}}, \bar{P}_{S}\right)
$$


where $\bar{P}_{S}$ and $I$ stand for maximum average transmit power available at the BS and ITC at $P_{D}$. In the first time, $\mathrm{R}$ received the following signal:

$$
y_{R}=h_{S R}\left(\sqrt{a_{1} P_{S}} x_{1}+\sqrt{a_{2} P_{S}} x_{2}\right)+n_{R}
$$

in which $n_{R}$ stand for the AWGN noise terms at R.

Then, we compute signal-to-interference-plus-noise ratio (SINR) and signal-to-noise ratio (SNR) of decoding $x_{1}$ and $x_{2}$ at $R$ and they can be respectively written as:

$$
\gamma_{R}^{1}=\frac{a_{1} \rho_{S}\left|h_{S R}\right|^{2}}{a_{2} \rho_{S}\left|h_{S R}\right|^{2}+1}
$$

and

$$
\gamma_{R}^{2}=a_{2} \rho_{S}\left|h_{S R}\right|^{2}
$$

where $\rho_{S}=\frac{P_{S}}{\sigma^{2}}$ is the transmit SNR at the BS.

In the second phase, the detected superimposed signal $\sqrt{\beta_{1} P_{R}} \bar{x}_{1}+\sqrt{\beta_{2} P_{R}} \bar{x}_{2}$ will be forwarded to destinations. It is noted that $P_{R}$ is the transmit power at $R$. The received signal at $D_{i}$ can be given as:

$$
y_{D i}=h_{R D i}\left(\sqrt{\beta_{1} P_{R}} \bar{x}_{1}+\sqrt{\beta_{2} P_{R}} \bar{x}_{2}\right)+n_{D i}, \forall i \in\{1,2\}
$$

where $n_{D i}$ is the AWGN noise terms at $D_{i}$. Further, due to the fact that $D_{1}$ is allocated with higher power factor, it can detect $\bar{x}_{1}$ by treating $\bar{x}_{2}$ as a background noise and it can be achieved following SINR.

$$
\gamma_{D 1}=\frac{\left|h_{R D 1}\right|^{2} \beta_{1} \rho_{R}}{\left|h_{R D 1}\right|^{2} \beta_{2} \rho_{R}+1}
$$

Furthermore, $D_{2}$ with SIC is required to detect $\bar{x}_{1}$ while $\bar{x}_{2}$ is considered as a noise. The SINR in this case can be expressed:

$$
\gamma_{D 2 \rightarrow 1}=\frac{\left|h_{R D 2}\right|^{2} \beta_{1} \rho_{R}}{\left|h_{R D 2}\right|^{2} \beta_{2} \rho_{R}+1}
$$

where $\rho_{R}=\frac{P_{R}}{\sigma^{2}}$ is the transmit SNR at $R$. the following SNR can be obtained to $D_{2}$ detects its own signal.

$$
\gamma_{D 2}=\left|h_{R D 2}\right|^{2} \beta_{2} \rho_{R}
$$

The signal received at $E$

$$
y_{E}=h_{R E}\left(\sqrt{\beta_{1} P_{E}} \bar{x}_{1}+\sqrt{\beta_{2} P_{E}} \bar{x}_{1}\right)+n_{E}
$$

where $n_{E}$ indicates the AWGN noise terms at $E . h_{R E}$ is the channel between Relay and $E$.

The signal-to-noise ratio (SNR) at $E$ :

$$
\gamma_{E i}=\beta_{i} \rho_{E}\left|h_{R E}\right|^{2}
$$


where $\rho_{E}=\frac{P_{E}}{\sigma^{2}}$ is transmit SNR at $E$. The achievable rates of $x_{1}$ and $x_{2}$ are derived respectively:

$$
\begin{aligned}
& K_{1}=\frac{1}{2} \log _{2}\left(1+\min \left(\gamma_{R}^{1}, \gamma_{D 2 \rightarrow 1}, \gamma_{D 1}\right)\right) \\
& K_{2}=\frac{1}{2} \log _{2}\left(1+\min \left(\gamma_{R}^{2}, \gamma_{D 2}\right)\right)
\end{aligned}
$$

and the achievable rates of eavesdropper's signal is given by:

$$
K_{E i}=\frac{1}{2} \log _{2}\left(1+\gamma_{E i}\right)
$$

the secrecy capacity for $D 1$ is obtained as:

$$
C_{1}=\left[\frac{1}{2} \log _{2}\left(\frac{1+\min \left(\gamma_{R}^{1}, \gamma_{D 2 \rightarrow 1}, \gamma_{D 1}\right)}{1+\gamma_{E 1}}\right)\right]^{+}
$$

the secrecy capacity for $D 2$ is obtained as:

$$
C_{2}=\left[\frac{1}{2} \log _{2}\left(\frac{1+\min \left(\gamma_{R}^{2}, \gamma_{D 2}\right)}{1+\gamma_{E 2}}\right)\right]^{+}
$$

where $[x]^{+}=\max \{x, 0\}$.

\section{SECRECY PERFORMANCE ANALYSIS}

In NOMA systems, with the help of a relay two signals are transmitted from the source to $D_{1}$ and $D_{2}$ respectively. As a result, outage happens when either $C_{1}$ or $C_{2}$ falls below their own target rates. Be exploiting this definition, the secure outage probability (SOP) can be formulated as following sections:

\subsection{SOP at D1}

$$
\begin{aligned}
P_{S O P 1}= & \operatorname{Pr}\left(C_{1}<R_{1}\right) \\
= & 1-\underbrace{\operatorname{Pr}\left(\frac{a_{1} \rho_{S}\left|h_{S R}\right|^{2}}{a_{2} \rho_{S}\left|h_{S R}\right|^{2}+1}>\theta\left|h_{R E}\right|^{2}+\omega_{1}\right)}_{A} \operatorname{Pr} \underbrace{\left(\frac{\left|h_{R D 2}\right|^{2} \beta_{1} \rho_{R}}{\left|h_{R D 2}\right|^{2} \beta_{2} \rho_{R}+1}>\theta\left|h_{R E}\right|^{2}+\omega_{1}\right)}_{B} \\
& \times \underbrace{\operatorname{Pr}(\underbrace{\left(\frac{\left|h_{R D 1}\right|^{2} \beta_{1} \rho_{R}}{\mid h_{R} \rho_{R}+1}>\theta\left|h_{R E}\right|^{2}+\omega_{1}\right)}_{R D 1}}_{C}
\end{aligned}
$$

where $\theta=2^{2 R_{1}} \beta_{1} \rho_{E}, \omega_{1}=2^{2 R_{1}}-1$.

Firstly, $A$ can be written by:

$$
A=\underbrace{\operatorname{Pr}\left(\left|h_{S R}\right|^{2} \geq \frac{\left(\theta\left|h_{R E}\right|^{2}+\omega_{1}\right)}{a_{1} \bar{\rho}_{S}-\left(\theta\left|h_{R E}\right|^{2}+\omega_{1}\right) a_{2} \bar{\rho}_{S}}, \bar{\rho}_{S}<\frac{\rho_{I}}{\left|h_{S P}\right|^{2}}\right)}_{A_{1}}+\underbrace{\operatorname{Pr}\left(\left|h_{S R}\right|^{2} \geq \frac{\left(\theta\left|h_{R E}\right|^{2}+\omega_{1}\right)\left|h_{S P}\right|^{2}}{\left(a_{1}-\left(\theta\left|h_{R E}\right|^{2}+\omega_{1}\right) a_{2}\right) \rho_{I}}, \bar{\rho}_{S}>\frac{\rho_{I}}{\left|h_{S P}\right|^{2}}\right)}_{A_{2}}
$$

we have $A_{1}$ as: 


$$
A_{1}=\operatorname{Pr}\left(\left|h_{S R}\right|^{2} \geq \frac{\theta\left|h_{R E}\right|^{2}+\omega_{1}}{a_{1} \bar{\rho}_{S}-\left(\theta\left|h_{R E}\right|^{2}+\omega_{1}\right) a_{2} \bar{\rho}_{S}},\left|h_{S P}\right|^{2}<\frac{\rho_{I}}{\bar{\rho}_{S}}\right)
$$

provided that $\left|h_{R E}\right|^{2}<\frac{a_{1}-a_{2} \omega_{1}}{a_{2} \theta}$, then $A_{1}$ can be calculated as:

$$
\begin{aligned}
A_{1} & =\int_{0}^{\frac{\rho_{I}}{\bar{\rho}_{S}}} f_{\left|h_{S P}\right|^{2}}(x) \int_{0}^{\frac{a_{1}-a_{2} \omega_{1}}{a_{2} \theta}} f_{\left|h_{R E}\right|^{2}}(y) \int_{\overline{a_{1} \bar{\rho}_{S}-\left(\theta y+\omega_{1}\right) a_{2} \bar{\rho}_{S}}}^{\infty} f_{\left|h_{S R}\right|^{2}}(z) d x d y d z \\
& =\left(1-e^{-\frac{\rho_{I}}{\overline{\bar{\rho}_{S}} \lambda_{S P}}}\right) \frac{1}{\lambda_{R E}} \int_{0}^{\frac{a_{1}-a_{1} a_{2}}{a_{2} \theta}} e^{-\frac{y}{\lambda_{R E}}} e^{-\frac{\theta y+\omega_{1}}{\left(a_{1}-c_{1} a_{2}-a_{2} \theta y\right) \bar{\rho}_{S} \lambda_{S R}}} d y
\end{aligned}
$$

where $u=a_{1}-\omega_{1} a_{2}, v=a_{2} \theta$, we have:

$$
A_{1}=\left(1-e^{-\frac{\rho_{I}}{\bar{\rho}_{S} \lambda_{S P}}}\right) \frac{1}{\lambda_{R E}} \int_{0}^{\frac{u}{v}} e^{-\frac{y}{\lambda_{R E}}} e^{-\frac{\theta y+\omega_{1}}{(u-v y) \bar{\rho}_{S} \lambda_{S R}}} d y
$$

let $u-v y=q \rightarrow y=\frac{u-q}{v} \Leftrightarrow d y=\frac{d q}{-v}$. We have:

$$
A_{1}=\left(1-e^{-\frac{\rho_{I}}{\overline{\bar{P}}_{S} \lambda_{S P}}}\right) \frac{e^{-\frac{u}{v \lambda_{R E}}+\frac{\theta}{v \bar{p}_{S} \lambda_{S R}}}}{v \lambda_{R E}} \zeta_{1}
$$

where $\zeta_{1}=\int_{0}^{u} e^{\frac{q}{\nu_{R E}}-\frac{\theta u+v Q_{1}}{v q \bar{p}_{S} \lambda_{S R}}} d q$

Similar $A_{1}$, we can calculated $A_{2}$ as:

$$
A_{2}=\frac{1}{v \lambda_{R E}} e^{-\frac{\rho_{I}}{\overline{\bar{P}}_{S} \lambda_{S P}}-\frac{u}{v \lambda_{R E}}+\frac{\theta \rho_{I}}{v \rho_{I} \bar{\rho}_{S} \lambda_{S R}}} \zeta_{2}
$$

where $\zeta_{2}=\int_{0}^{u} \frac{q v \rho_{I} \lambda_{S R}}{q v \rho_{I} \lambda_{S R}+\lambda_{S P}\left(\theta u-\theta q+v \omega_{1}\right)} e^{\frac{q}{v \lambda_{R E}}-\frac{\left(\theta u+v \omega_{1}\right) \rho_{I}}{v q \rho_{I} \bar{\rho}_{S} \lambda_{S R}}} d q$

From (17) and (18) we find $A$ by:

$$
A=\left(1-e^{-\frac{\rho_{l}}{\overline{\bar{\rho}_{S}} \lambda_{S P}}}\right) \frac{e^{-\frac{u}{v \lambda_{R E}}+\frac{\theta}{v \overline{\bar{S}_{S}} \lambda_{S R}}}}{v \lambda_{R E}} \zeta_{1}+\frac{1}{v \lambda_{R E}} e^{-\frac{\rho_{l}}{\overline{\bar{\rho}_{S}} \lambda_{S P}}-\frac{u}{v \lambda_{R E}}+\frac{\theta \rho_{l}}{v \rho_{l} \overline{\bar{S}}_{S} \lambda_{S R}}} \zeta_{2}
$$

the secondly, B can be written as:

$$
B=\operatorname{Pr}\left(\left|h_{R D 2}\right|^{2}>\frac{\theta\left|h_{R E}\right|^{2}+\omega_{1}}{\left(\beta_{1}-\omega_{1} \beta_{2}-\beta_{2} \theta\left|h_{R E}\right|^{2}\right) \rho_{R}}\right)
$$


provided that $\left|h_{R E}\right|^{2}<\frac{\beta_{1}-\omega_{1} \beta_{2}}{\theta \beta_{2}}, B$ calculated as:

$$
B=\int_{0}^{\frac{\beta_{1}-\omega_{1} \beta_{2}}{\theta \beta_{2}}} f_{\left|h_{R E}\right|^{2}}(x) \int_{\frac{\theta x+\omega_{1}}{\left(\beta_{1}-\omega_{1} \beta_{2}-\beta_{2} \theta x\right) \rho_{R}}}^{\infty} f_{\left|h_{R D D}\right|^{2}}(y) d x d y=\frac{1}{\lambda_{R E}} \int_{0}^{\frac{\beta_{1}-\omega_{1} \beta_{2}}{\theta \beta_{2}}} e^{-\frac{x}{\lambda_{R E}}} e^{-\frac{\theta x+\omega_{1}}{\left(\beta_{1}-\omega_{1} \beta_{2}-\beta_{2} \theta x\right) \rho_{R} \lambda_{R D 2}}} d x
$$

where $n=\beta_{1}-\omega_{1} \beta_{2}, m=\theta \beta_{2}$, we have:

$$
B=\frac{1}{\lambda_{R E}} \int_{0}^{\frac{n}{m}} e^{-\frac{x}{\lambda_{R E}}} e^{-\frac{\theta x+\omega_{1}}{(n-m x) \rho_{R} \lambda_{R D 2}}} d x
$$

let $t=n-m x \Leftrightarrow x=\frac{n-t}{m} \Leftrightarrow d x=\frac{d t}{-m}$. We have:

$$
B=\frac{e^{-\frac{n}{m \lambda_{R E}}+\frac{\theta}{m \rho_{R} \lambda_{R D 2}}}}{m \lambda_{R E}} \vartheta_{1}
$$

where $\vartheta_{1}=\int_{0}^{n} e^{\frac{t}{m \lambda_{R E}}} e^{-\frac{\theta n+m \omega_{1}}{m t \rho_{R} \lambda_{R D 2}}} d t$.

Similar (20), we can calculated $\mathrm{C}$ as:

$$
C=\frac{e^{-\frac{n}{m \lambda_{R E}}+\frac{\theta}{m \rho_{R} \lambda_{R D 1}}}}{m \lambda_{R E}} \vartheta_{2}
$$

where $\vartheta_{1}=\int_{0}^{n} e^{\frac{t}{m \lambda_{R E}}-\frac{\theta n+m Q_{1}}{m t \rho_{R} \lambda_{R D 1}}} d t$

From (19), (20) and (21) we calculated $P_{S O P 1}$ as:

$$
\begin{aligned}
& P_{S O P 1}=1-\left[\left(1-e^{-\frac{\rho_{I}}{\bar{\rho}_{S} \lambda_{S P}}}\right) \frac{e^{-\frac{u}{v \lambda_{R E}}+\frac{\theta}{v \bar{\nu} \lambda_{S R}}}}{v \lambda_{R E}} \zeta_{1}+\frac{1}{v \lambda_{R E}} e^{-\frac{\rho_{I}}{\overline{\bar{S}}_{S} \lambda_{S P}}-\frac{u}{v \lambda_{R E}}+\frac{\theta \rho_{I}}{v \rho_{I} \overline{\bar{S}}_{S R} \lambda_{S R}}} \zeta_{2}\right] \\
& \left(\frac{e^{-\frac{n}{m \lambda_{R E}}+\frac{\theta}{m \rho_{R} \lambda_{R D 2}}}}{m \lambda_{R E}} \vartheta_{2}\right)\left(\frac{e^{-\frac{n}{m \lambda_{R E}}+\frac{\theta}{m \rho_{R} \lambda_{R D 1}}}}{m \lambda_{R E}} \vartheta_{1}\right)
\end{aligned}
$$

\subsection{SOP at D2}

By definition, we have SOP as:

$$
\begin{aligned}
P_{S O P 2} & =\operatorname{Pr}\left(C<R_{2}\right) \\
& =1-\underbrace{\operatorname{Pr}\left(a_{2} \rho_{S}\left|h_{S R}\right|^{2}>\psi\left|h_{R E}\right|^{2}+\omega_{2}\right)}_{D} \underbrace{\operatorname{Pr}\left(\beta_{2} \rho_{R}\left|h_{R D 2}\right|^{2}>\psi\left|h_{R E}\right|^{2}+\omega_{2}\right)}_{E}
\end{aligned}
$$

where $\psi=2^{2 R_{2}} \beta_{2} \rho_{E}, \omega_{2}=2^{2 R_{2}}-1$. Firstly, $D$ can be rewritten as:

$$
D=\underbrace{\operatorname{Pr}\left(a_{2} \bar{\rho}_{S}\left|h_{S R}\right|^{2}>\psi\left|h_{R E}\right|^{2}+\omega_{2},\left|h_{S P}\right|^{2}<\frac{\rho_{I}}{\bar{\rho}_{S}}\right)}_{D_{1}}+\underbrace{\operatorname{Pr}\left(\frac{a_{2} \rho_{I}\left|h_{S R}\right|^{2}}{\left|h_{S P}\right|^{2}}>\psi\left|h_{R E}\right|^{2}+\omega_{2},\left|h_{S P}\right|^{2}>\frac{\rho_{I}}{\bar{\rho}_{S}}\right)}_{D_{2}}
$$


let $\bar{a}=\frac{1}{a_{2} \bar{\rho}_{S}}, D_{1}$ can be computed as:

$$
D_{1}=\operatorname{Pr}\left(\left|h_{S R}\right|^{2}>\psi \bar{a}\left|h_{R E}\right|^{2}+\omega_{2} \bar{a},\left|h_{S P}\right|^{2}<\frac{\rho_{I}}{\bar{\rho}_{S}}\right)=\left(1-e^{-\frac{\rho_{I}}{\lambda_{S P} \bar{\rho}_{S}}}\right) e^{-\frac{\omega_{2} \bar{a}}{\lambda_{S R}}}\left(\frac{\lambda_{R E} \psi \bar{a}}{\lambda_{S R}}+1\right)^{-1}
$$

next, let $\tilde{a}=\frac{1}{a_{2} \rho_{I}}, D_{2}$ can calculated as:

$$
\begin{aligned}
D_{2} & =\operatorname{Pr}\left(\left|h_{S R}\right|^{2}>\psi \tilde{a}\left|h_{S P}\right|^{2}\left|h_{R E}\right|^{2}+\omega_{2} \tilde{a}\left|h_{S P}\right|^{2},\left|h_{S P}\right|^{2}>\frac{\rho_{I}}{\bar{\rho}_{S}}\right) \\
& =e^{-\frac{\rho_{I}}{\lambda_{S P} \bar{\rho}_{S}}-\frac{\rho_{l} \omega_{2} \tilde{a}}{\lambda_{S R} \bar{\rho}_{S}}} \frac{\lambda_{S R}}{\psi \lambda_{R E} \lambda_{S P} \tilde{a}} \int_{0}^{\infty} \frac{e^{-x\left(\frac{1}{\lambda_{R E}}+\frac{\rho_{I} \psi \tilde{a}}{\lambda_{S R} \bar{\rho}_{S}}\right)}}{\left(x+\frac{\lambda_{S R}+\lambda_{S P} \omega_{2} \tilde{a}}{\psi \lambda_{S P} \tilde{a}}\right)} d x
\end{aligned}
$$

Let $\beta=\frac{\lambda_{S R}+\lambda_{S P} \omega_{2} \tilde{a}}{\psi \lambda_{S P} \tilde{a}}, \mu=\left(\frac{1}{\lambda_{R E}}+\frac{\rho_{I} \psi \tilde{a}}{\lambda_{S R} \bar{\rho}_{S}}\right)$, we have $D_{2}$

$$
D_{2}=e^{-\frac{\rho_{I}}{\lambda_{S P} \bar{\rho}_{S}}-\frac{\rho_{1} \omega_{2} \tilde{a}}{\lambda_{S R} \bar{P}_{S}}} \frac{\lambda_{S R}}{\psi \lambda_{R E} \lambda_{S P} \tilde{a}} \int_{0}^{\infty} \frac{e^{-x \mu}}{(x+\beta)} d x=e^{-\frac{\rho_{I}}{\lambda_{S P} \bar{P}_{S}}-\frac{\rho_{1} \omega_{2} \tilde{a}}{\lambda_{S R} \bar{\rho}_{S}}} \frac{\lambda_{S R}}{\psi \lambda_{R E} \lambda_{S P} \tilde{a}} e^{\beta \mu} E_{1}(\mu \beta)
$$

from (22) and (23) $D$ can be as:

$$
D=\left(1-e^{-\frac{\rho_{I}}{\lambda_{S P} \bar{\rho}_{S}}}\right) e^{-\frac{\omega_{2} \bar{a}}{\lambda_{S R}}}\left(\frac{\lambda_{R E} \psi \bar{a}}{\lambda_{S R}}+1\right)^{-1}+e^{-\frac{\rho_{l}}{\lambda_{S P} \bar{\rho}_{S}}-\frac{\rho_{I} \omega_{2} \tilde{a}}{\lambda_{S R} \bar{\rho}_{S}}} \frac{\lambda_{S R}}{\psi \lambda_{R E} \lambda_{S P} \tilde{a}} e^{\beta \mu} E_{1}(\mu \beta)
$$

next, with $\bar{b}=\frac{1}{\beta_{2} \rho_{R}}$ E can be calculated as:

$$
E=\int_{0}^{\infty} f_{\left|h_{R E}\right|^{2}}(x) \int_{\psi \bar{b} x+\omega_{2} \bar{b}}^{\infty} f_{\left|h_{R D 2}\right|^{2}}(y) d x d y=\frac{\lambda_{R D 2}}{\psi \bar{b} \lambda_{R E}+\lambda_{R D 2}} e^{-\frac{\omega_{2} \bar{b}}{\lambda_{R D 2}}}
$$

from (24) and (25) it can be calculated $P_{S O P 2}$ as:

$$
\begin{aligned}
& P_{S O P 2}=1-\frac{\lambda_{R D 2}}{\psi \bar{b} \lambda_{R E}+\lambda_{R D 2}} e^{-\frac{\omega_{2} \bar{a}}{\lambda_{S R}}-\frac{\omega_{2} \bar{b}}{\lambda_{R D 2}}}\left(1-e^{-\frac{\rho_{1}}{\lambda_{S P} \bar{\rho}_{S}}}\right)\left(\frac{\lambda_{R E} \psi \bar{a}}{\lambda_{S R}}+1\right)^{-1} \\
& -\frac{\lambda_{S R} \lambda_{R D 2}}{\psi \lambda_{R E} \lambda_{S P} \tilde{a}\left(\psi \bar{b} \lambda_{R E}+\lambda_{R D 2}\right)} e^{-\frac{\rho_{1}}{\lambda_{S P} \bar{\rho}_{S}}-\frac{\rho_{I} \omega_{2} \tilde{a}}{\lambda_{S R} \bar{\rho}_{S}}-\frac{\omega_{2} \bar{b}}{\lambda_{R D 2}}} e^{\beta \mu} E_{1}(\mu \beta)
\end{aligned}
$$

\section{NUMB ER RESULTS}

This section of this study represents numerical and simulation results for the SOP over related Rayleigh fading channels. The adopted system parameters are given in each figure. We evaluate the impact of target rates on the secrecy outage performance for NOMA-based cognitive radio as in Figure 2. Higher target rates result in worse SOP performance. It can be seen performance gap among two signals is large at $R_{1}=R_{2}=0.5$. The reason is that different power allocation factors for each signal leads to different SOP 
performance. In other observation, impact of interference $\rho_{I}=10,15,20(d B)$ to secure performance of two signals can be seen as Figure 3. It can be confirmed lower interference provides better secure performance. Such performance gap will be reduced at $R_{1}=R_{2}=1$. The analytical values of the OP for $x_{1}, x_{2}$ with various target rates and SNR threshold values are shown in Figure 4. It is observed that curves of the OP improve by increasing the transmit SNR. It is worth noting that no ICT case exhibits better secure performance.

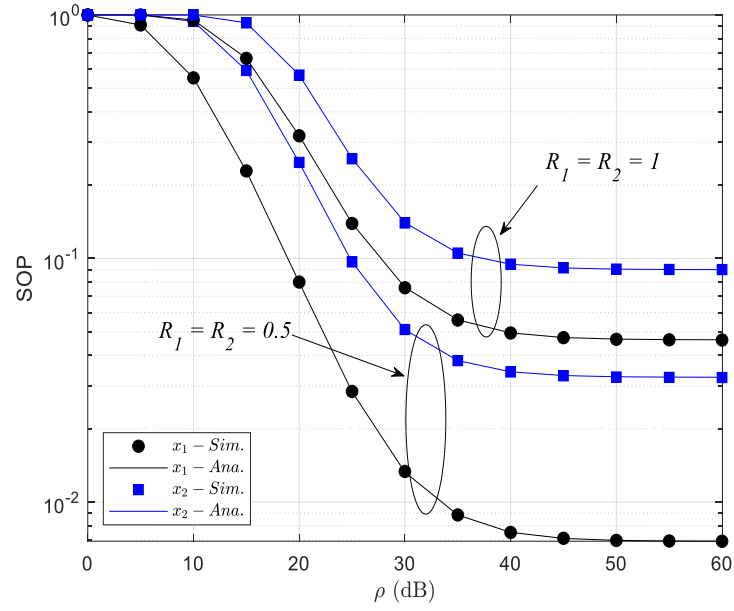

Figure 2. $a_{1}=\beta_{1}=0.9, a_{2}=\beta_{2}=0.1, \rho_{I}=15 d B$, $\rho_{E}=5 d B, \lambda_{S P}=0.1, \lambda_{S R}=1, \lambda_{R D 1}=0.9, \lambda_{R D 2}=0.6$ and $\lambda_{R E}=0.1$

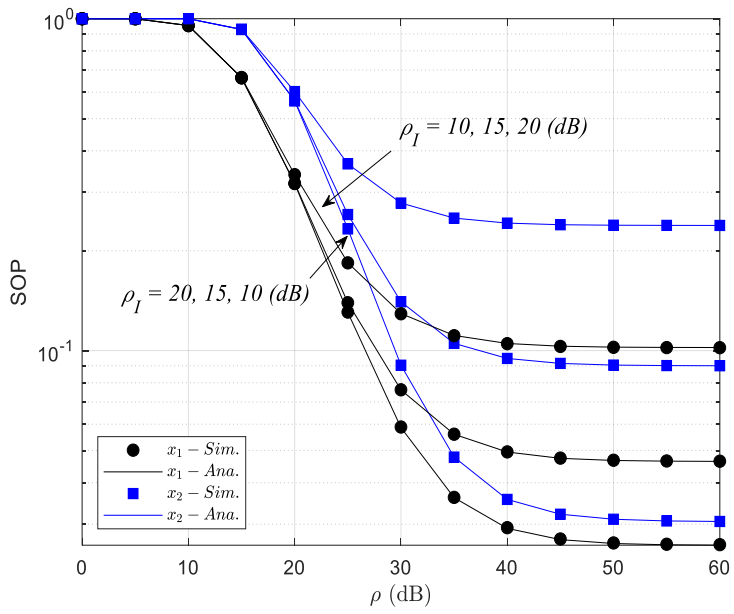

Figure 3. SOP versus trans mit SNR with different

$$
\begin{gathered}
\rho_{I}\left(a_{1}=\beta_{1}=0.9, a_{2}=\beta_{2}=0.1, \rho_{E}=5 \mathrm{~dB},\right. \\
\lambda_{S P}=0.1, \lambda_{S R}=1, \lambda_{R D 1}=0.9, \lambda_{R D 2}=0.6 \\
\text { and } \left.\lambda_{R E}=0.1\right)
\end{gathered}
$$

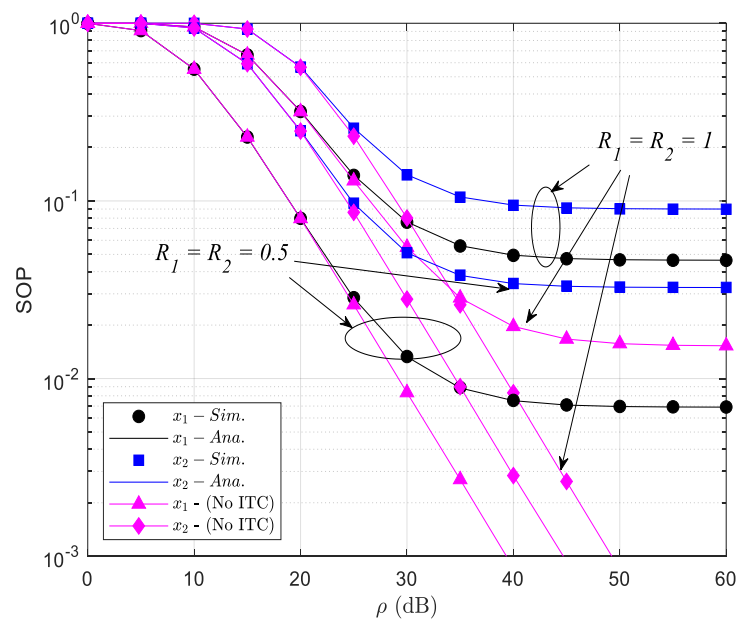

Figure 4. SOP versus transmit SNR as ITC consideration $\left(a_{1}=\beta_{1}=0.9, a_{2}=\beta_{2}=0.1, \rho_{I}=15 d B\right.$,

$$
\left.\rho_{E}=5 \mathrm{~dB}, \lambda_{S P}=0.1, \lambda_{S R}=1, \lambda_{R D 1}=0.9, \lambda_{R D 2}=0.6 \text { and } \lambda_{R E}=0.1\right)
$$

\section{CONCLUSION}

In this paper, in order to evaluate security performance of CR-NOMA systems, SOP performance in condition of fixed power allocation scheme is examined. A limitation of transmit power at the secondary in CR-NOMA results in varying secure performance. The threshold data rates and impact of interference from the primary network are main factors affecting to systemperformance. 


\section{REFERENCES}

[1] Z. Ding, X. Lei, G. K. Karagiannidis, "A survey on non-orthogonal multiple access for 5G networks: Research challenges and future trends IEEE," J. Sel. Areas Commun, vol. 35, no. 10, pp. 2181-2195, 2017.

[2] Y. Liu, Z. Qin, M., "Elkashlan. Nonorthogonal multiple access for 5G and beyond," Proceedings of the IEEE, vol. 105, no. 12, pp. 2347-2381, 2017.

[3] Z. Zhang, H. Sun, and R. Q. Hu, "Downlink and uplink non-orthogonal multiple access in a dense wireless network," IEEE J. Sel. AreasCommun, vol. 35, no. 12, pp. 2771-2784, 2017.

[4] Tan N. Nguyen, Minh Tran, Van-Duc Phan, H.-N. Nguyen and T.-L. Nguyen,"Half-duplex power beacon-assisted energy harvesting relaying networks: system performance analysis," International Journal of Electrical and Computer Engineering, vol. 9, no. 5, pp. 3399-3406, Oct. 2019.

[5] Tan N. Nguyen, Van-Duc Phan, H.-N. Nguyen, Minh Tran and T.-T. Tran,"Performance Analysis for PowerSplitting Energy Harvesting Based Two-Way Full-Duplex Relaying Network over Nakagami-m Fading Channel," Telecommunication, Computing, Electronics and Control TELKOMNIKA, vol. 17, no. 4, pp.1595-1603, Aug. 2019.

[6] Phu Tran Tin, Minh Tran, Tan N.Nguyen and Tran Thanh Trang,"Energy Harvesting Half-Duplex AF Power Splitting Protocol Relay Network over Rician Channel in Case of Maximizing Capacity", Telecommunication, Computing, Electronics and Control TELKOMNIKA, vol. 17, no. 4, pp. 1615-1624, Aug. 2019.

[7] Phu Tran Tin, Minh Tran, Tan N. Nguyen, Thanh-Long Nguyen,"System performance analy sis of hybrid timepower switching protocol of EH bidirectional relaying network in amplify -and-forward mode", Indonesian Journal of Electrical Engineering and Computer Science, vol. 14, no. 1, pp. 118-126, Apr. 2019.

[8] Dinh-Thuan Do and M. S., Van Nguyen, "Device-to-device transmission modes in NOMA network with and without Wireless Power Transfer," Computer Communications, vol. 139, pp. 67-77, May 2019.

[9] D.-T. Do and A.-T. Le, "NOMA based cognitive relaying: Transceiver hardware impairments, relay selection policies and outage performance comparison," Computer Communications, vol. 146, pp. 144-154, 2019.

[10] D.-T. Do, M. Vaezi and T.-L. Nguyen, "Wireless Powered Cooperative Relaying using NOM A with Imperfect CSI," in Proc. of IEEE Globecom Workshops (GC Wkshps), Abu Dhabi, UAE, pp. 1-6, 2018.

[11] T.-L. Nguyen and Dinh-Thuan Do, "Exploiting Impacts of Intercell Interference on SWIPT-assisted Nonorthogonal Multiple Access," Wireless Communications and Mobile Computing, vol. 2018, pp. 1-12, 2018

[12] D.-T. Do, A.-T. Le, C.-B. Le and B. M. Lee "On Exact Outage and Throughput Performance of Cognitive Radio based Non-Orthogonal Multiple Access Networks with and Without D2D Link," Sensors (Basel), vol. 19, no. 15, pp. 1-17, 2019.

[13] H. Zhang, B. Wang, C. Jiang, "Energy efficient dynamic resource optimization in NOMA sy stem," IEEE Trans. Wireless Commun, vol. 17, no. 9, pp.5671-5683, 2018.

[14] Y. Deng, L. Wang, S. A. R. Zaidi, "Artificial-noise aided secure transmission in large scale spectrum sharing networks," IEEE Trans. Wireless Commun, vol. 64, no. 5, pp. 2116-2129, 2016.

[15] Y. Liu, Z. Ding, M. Elkashlan, "Non-orthogonal multiple access in large-scale underlay cognitive radio networks," IEEE Trans. Veh.Technol, vol. 65, no. 12, pp. 152-157, 2016.

[16] Phu Tran Tin, Le Anh Vu, Tan N. Nguyen, Thanh-Long Nguyen, "User selection protocol in DF cooperative networks with hybrid TSR-PSR protocol based full-duplex energy harvesting over Ray leigh fading channel: sy stem performance analysis," Indonesian Journal of Electrical Engineering and Computer Science, vol. 13, no. 2, pp. 534-542, Feb. 2019.

[17] Tan N. Nguyen, Minh Tran, D.-H. Ha, T. Tran and M. Voznak,"Multi-Source in DF Cooperative Networks With PSR protocol Based Full-Duplex Energy Harvesting Over Rayleigh Fading Channel: Performance Analy sis," Proceedings of the Estonian Academy of Sciences, vol. 68, no. 3, pp. 264-275, May. 2019. (SCIE, IF 0.51-2018.

[18] Tan N. Nguyen, T. H. Q.M inh, T.-L. Nguyen, D.-H. Ha and M. Voznak, "Performance Analysis of a User Selection Protocol in Cooperative Networks with Power Splitting Protocol Based Energy Harvesting Over Nakagami-m/Rayleigh Channel", Electronics, vol. 8, no. 4, art. no. 448, Apr. 2019.

[19] T. N. Nguyen, M. Tran, T. Nguyen and M. Voznak, "Adaptive relaying protocol for decode and forward full-duplex system over Rician fading channel: System performance analy sis," in China Communications, vol. 16, no. 3, pp. 92-102, March 2019.

[20] Tan N. Nguyen, T. H. Q. Minh, T.-L. Nguyen, D.-H. Ha and M. Voznak," Multi-Source Power Splitting Energy Harvesting Relaying Network In Half-Duplex System Over Block Rayleigh Fading Channel: Sy stem Performance Analy sis", Electronics, vol. 8, no. 1, Art. no. 67, Jan 2019.

[21] Tan N. Nguyen, M. Tran, P. T. Tran, T. Phu, T.-L. Nguyen, D.-H. Ha and M. Voznak, "On the Performance of Power Splitting Energy Harvested Wireless Full-Duplex Relaying Network with Imperfect CSI over Dissimilar Channels," Security and Communication Networks, Vol.2018, 11 pages, Art.no.6036087, Jan. 2019.

[22] J. Chen, L. Yang, and M.-S. Alouini, "Physical layer security for cooperative NOMA systems," IEEE Trans. Veh. Technol, vol. 67, no. 5, pp. 4645-4649, 2018.

[23] F. Zhou, Z. Chu, H. Sun, R. Q. Hu, and L. Hanzo, "Artificial Noise Aided Secure Cognitive Beamforming for Cooperative MISO-NOM A Using SWIPT,” IEEE J. Sel. Areas Commun, vol. 36, no. 4, pp.918-931, 2018

[24] Z. Yang, J. A. Hussein, P. Xu, Z. Ding, and Y. Wu, "Power Allocation Study for Non-Orthogonal Multiple Access Networks with Multicast Unicast Transmission," IEEE Trans. Wirel. Commun, vol. 17, no. 6, pp. 3588-3599, 2018.

[25] L. Xu, A. Nallanathan, X. Pan, J. Yang, and W. Liao, "Security-Aware Resource Allocation with Delay Constraint for NOM A-Based Cognitive Radio Network," IEEE Trans. Inf. Forensics Secure, vol. 13, no. 2, pp. 366-376, 2018. 